\title{
TECNOLOGÍA Y EMPLEO
}

\author{
Sofía Méndez Villarreal * \\ El Colegio de México
}

\section{INTRODUCCIÓN}

El INTERÉs por estudiar los problemas de la tecnología en los países de escaso desarrollo deriva del papel paradójico que parece tener en el desarrollo económico y social de estos países. En efecto, la influencia de los avances científicos y tecnológicos ha sido altamente positiva en el crecimiento de los países industrializados. Sin embargo, la forma en que este conocimiento ha sido incorporado en las áreas no desarrolladas ha dado origen a una serie de dudas sobre su relevancia y adecuación a las condiciones de los países atrasados.

Entre las repercusiones negativas asociadas a la tecnología en los países menos desarrollados se han destacado: la disociación entre el tipo de tecnología utilizada y la dotación de recursos y factores locales; las condiciones en que tiene lugar el proceso de comercialización de tecnología; la implantación de ciertos patrones de consumo que no corresponden a las posibilidades y niveles de ingreso de los países en desarrollo; el escaso estímulo a la integración vertical del proceso productivo en los países receptores de teconología, etc. Todos estos son aspectos que se explican en parte por la reducida capacidad para seleccionar y adaptar la tecnología importada en función de los objetivos económicos y sociaes locales.

En el presente ensayo se intenta un examen de las repercusiones del tipo de tecnología utilizada en el sector industrial sobre dos aspectos principales: la estructura productiva, reflejada en la combinación de bienes que se producen; y la combinación de diferentes procesos tecnológicos, reflejada en las distintas proporciones factoriales (capital y trabajo) que se utilizan en un mismo sector productivo. La idea subyacente en este planteamiento es que las posibilidades de generación de empleo de la economía están íntimamente ligadas tanto a la estrategia de desarrollo sectorial como al tipo de tecnología que predomina en cada uno de los sectores.

La explicación acerca de la inadecuación de la tecnología en los países atrasados no se reduce a la simple hipótesis de que es "muy intensiva en capital". Para empezar, esta hipótesis no ha sido suficientemente probada para todos los sectores económicos. Incluso hay evidencia, basada en comparaciones del grado de intensidad de capital en sectores industriales específicos, que muestra que el uso de tecnologías menos intendístico.

* Agradezco a Noé Montealegre su ayuda en la preparación del material esta- 
sivas en capital es característica de los países menos desarrollados. ${ }^{1}$ Esto, no significa que no pueda haber industrias en los países subdesarrollados que al parecer estén excesivamente capitalizadas debido a que operan con una gran proporción de capacidad ociosa, pero éste es otro problema, que puede o no tener raíces tecnológicas.

Por otro lado, la mera existencia de una gran proporción de mano de obra desempleada y subempleada no es, por sí misma, evidencia del uso de excesiva intensidad de capital. Existe el peligro, además, de que aceptar a priori esta hipótesis conduciría a sugerir la solución simplista y mecánica de que debe promoverse, como objetivo en sí mismo, la introducción de tecnologías intensivas en mano de obra.

Una de las cuestiones que deben tenerse en cuenta en el análisis de la relación entre tecnología y empleo es que puede haber sectores que aparecen como "intensivos en mano de obra", debido a que sus relaciones capital-trabajo son relativamente bajas, pero que no necesariamente tienen una alta capacidad de absorción de mano de obra, desde el punto de vista dinámico. De ahí la importancia de examinar los efectos indirectos derivados de la expansión de los distintos sectores.

Entre los objetivos del análisis empírico que se presenta a continuación está la obtención de indicadores acerca del tipo de tecnologías que se asocia a las distintas industrias, y de su capacidad para generar empleo desde el punto de vista dinámico. Teniendo en cuenta las limitaciones de los indicadores y de la calidad de la información estadística disponible, los resultados constituyen apenas una primera aproximación al estudio de la estructura tecnológica y sus repercusiones sobre el empleo. A partir de la información disponible, referida esencialmente a los datos censales, es posible hacer algunas aproximaciones a un nivel de agregación intermedia, cuya utilidad principal consiste en detectar grupos de industrias en las que parezca fructífera la realización de esfuerzos de investigación posteriores. Idealmente, para el análisis de los problemas de tecnología se necesita disponer de información acerca de la estructura productiva de las empresas a nivel microeconómico.

Al analizar la conexión entre la tecnología y los problemas de empleo, es de suma importancia evitar el hacer planteamientos de tipo estático, tales como los que se derivan del simple análisis de las proporciones factoriales. Aunque el estudio de estas proporciones puede ser útil en una primera etapa; no parece muy fructífero plantear el problema y las posibles soluciones en términos puramente de tecnologías "intensivas en capital" o en "mano de obra".

El mero análisis de las proporciones factoriales resulta limitado para entender las raíces del problema, así como para formular acciones de política concretas.

En el planteamiento de los problemas de empleo hay que tener en cuenta dos consideraciones principales: 1) en principio, hay dificultad para dar respuesta precisa a la pregunta de si ha sido el uso de tecnolo-

1 Véanse, por ejemplo, las estimaciones utilizadas por E. L. Bacha en "Comparación entre la productividad industrial de México y los Estados Unidos", El Trimestre Económico, Núm. 132, 1966, cuadro 3; "Capital-Labour Ratios of Certain Industries in Some Countries; a Progress Report", Netherlands Economic Institute, 1955; y M. Mehta, "Capital Intensity of Manufacturing Industries in Some Selected Countries in the ECAFE Region", Asian Institute for Economic Development and Planning, Bangkok, 1969. 
gías intensivas en capital una de las principales causas del problema de empleo en las áreas de menor desarrollo relativo; y 2) el problema de empleo en estos países no se manifiesta en la forma de desempleo abierto, sino principalmente de subempleo, reflejado en el bajísimo nivel de productividad y retribución de una gran proporción de la fuerza de trabajo. De manera que la superación de los problemas de empleo equivale en mucho al objetivo de aumentar el nivel de calificación, la posición en el trabajo, y por ende la retribución de los trabajadores.

Considerado el problema de empleo en esta perspectiva, el objetivo de crear fuentes de trabajo para la mano de obra no calificada no constiuye más que una meta de corto plazo. En el plazo medio y en el largo plazo la posibilidad de solucionar el problema del empleo de mano de obra en un país como México dependerá de la generación de oportunidades de empleo que impliquen cierto grado de habilidad, así como de la formación de cuadros técnicos con un nivel de calificación intermedia.

En lo que se refiere a la tecnología, el problema se ha planteado esencialmente en términos de que es inadecuada en relación con la dotación de recursos y factores locales. En los enfoques convencionales más comúnmente utilizados se ha centrado la atención en la cuestión de las proporciones factoriales entre capital y mano de obra. Se dice que la tecnología utilizada en los países en vía de desarrollo es inadecuada debido a la influencia de distorsiones en los precios relativos de los factores, las cuales favorecen la utilización del factor capital y encarecen "artificialmente" el costo de la mano de obra. De aquí se deduce que si ia combinación de precios relativos se modificara en el sentido de disminuir el costo relativo de la mano de obra y aumentar el del capital se propiciaría la introducción de tecnologías más adecuadas a la proporción de factores locales que caracteriza a las áreas menos desarrolladas. ${ }^{2}$

Debe notarse que el énfasis en los efectos de las distorsiones de precios relativos involucra el juicio implícito de que las tecnologías adecuadas para los países en desarrollo serían aquellas intensivas de mano de obra, puesto que se supone que en estos países hay abundancia de mano de obra y escasez de capital.

En el planteamiento anterior hay que cuestionar varias de las premisas: ¿es el capital invertible un factor realmente escaso? ihay excedente del tipo de mano de obra requerido en los procesos productivos? ¿ha sido la selección tecnológica efectivamente un reșutado de los precios relativos de los factores?

En relación con los factores que influyen en el tipo de tecnología que se adopta se sabe poco, aun en lo que se refiere a los países industrializados. Las hipótesis hasta ahora elaboradas no son concluyentes en la explicación de la dirección, y tendencia del progreso tecnológico. Debe tenerse en cuenta además que la influencia de los precios relativos es sólo un factor entre muchos, de modo que la tendencia a singularizar el impacto de los precios relativos en la selección de tecnologías puede resultar engañosa. Esto no significa que el enfoque esté equivocado, ya que

2 W. Galenson, "The Employment Problems of Less Developed Countries: an Introduction", pp. 8-9 en Essays on Employment, OIT, Ginebra, 1971; Towards Full-employment: a Programme for Colombia, pp. 162-163, OIT, Ginebra, 1970; S. Jackson, Economically Appropriate Technologies for Developing Countries: a Survey, p. 24, Ocasional Paper, Serie No. 3, Overseas Development Council, Washington, D. C. 
en términos generales los argumentos son lógicos y parecen operar en alguna forma. Las reservas surgen en relación con su grado de influencia en la selección de procesos tecnológicos específicos. Debe además tenerse en cuenta que las imprfecciones del mercado y las dificultades para sustituir factores son relativamente mayores en el caso de los países atrasados económicamente. De manera .que el margen de selección tecnológica en estos países es considerablemente más limitado que en los países desarrollados.

Existen por otro lado explicaciones que en cierta forma se contraponen al argumento que enfatiza la influencia de las distorsiones de los precios relativos sobre la tecnología, tales como que: "la desigual distribución de la riqueza en un país como México constituye un obstáculo a la investigación científica y al adelanto tecnológico, porque los salarios no presionan a una utilización mayor de capital y tecnología moderna".3

Tanto el argumento sobre las distorsiones de precios relativos de los factores que (al no corresponder a su "costo social" o "precio sombra") conducen a la adopción de tecnologías que resultan demasiado intensivas en capital en relación con la dotación local de factores; como el argumento, probablemente más realista, de que el bajo costo de los salarios desalienta la investigación de tecnologías más modernas que impliquen una mayor utilización de capital, muestran cierta aceptación implícita de la influencia de los precios relativos en la selección de tecnologías.

Sin embargo, el hecho de que las conclusiones acerca de la dirección en que operan los precios relativos de los factores sobre el cambio tecnológico estén en franca contradicción, muestra el escaso conocimiento que existe en nuestros países acerca de los factores que influyen en la selección de tecnologías.

En relación con el supuesto de que la producción de ciertos bienes se asocia con determinada tecnología, se suele decir que la producción de bienes de capital y de consumo duraderos involucran el uso de tecnologías intensivas en capital, en tanto que la producción de bienes de consumo no duraderos se caracteriza por el uso de tecnologías intensivas en mano de obra. Con base en este punto de vista se han elaborado hipótesis tendientes a relacionar los conceptos de: distribución del ingrēso, tecnología y empleo. En esta forma se intenta explicar la existencia de mano de obra desempleada con base en el argumento de que la distribución del ingreso que caracteriza a los países subdesarrollados favorece la formación de patrones de consumo muy diversificados en los grupos de clase media y alta, lo cual tiene como consecuencia el florecimiento y expansión de las industrias productoras de bienes de consumo duraderos, los cuales se caracterizan por su alta intensidad de capital.

Este razonamiento tiene lógica y cierta validez. Sin embargo, es limitado por dos razones: primera, hace falta confirmar el supuesto sobre la intensidad factorial asociada a la producción de los distintos bienes; y segunda, la explicación es incompleta, ya que se soslaya el examen del grado de integración vertical en las distintas industrias, lo que permitiría considerar también las repercusiones en términos de efectos indirectos sobre el empleo.

Para resumir: de acuerdo con las consideraciones anteriores no sería

3 H. Flores de la Peña, "La educación superior y la investigación científica", El perfil de México 1980, México, Siglo XXI Editores, Vol. 2, 1970, p. 212. 
válido interpretar el problema del empleo como un resultado directo del tipo de tecnología utilizada, sino más bien como una manifestación del tipo de desarrollo que se ha seguido, el cual se ha caracterizado por una creciente extensión horizontal del aparato productivo, y por un reducido grado de eslabonamiento intersectorial. En el sector de manufacturas, en particular, las industrias ligeras han ido perdiendo dinamismo y por lo tanto capacidad de generación de empleo, al mismo tiempo que una gran proporción de las industrias intermedias y de capital aún no logran un grado de integración vertical local satisfactorio.

Como antes se mencionó, para analizar la estructura tecnológica de las diferentes industrias se utilizan como indicadores los coeficientes capital/trabajo y capital/producto. Sin embargo, al interpretar el nivel de estos coeficientes deben tenerse en cuenta no sólo las limitaciones de los distintos métodos de estimación de los conceptos de capital y trabajo, sino la influencia de distorsiones derivadas del diferente grado de utilización del capital instalado. Por ejemplo, existe la posibilidad de que las industrias ligeras aparezcan con indicadores que reflejarían al uso aparente de tecnologías "más intensivas en capital", debido a que la existencia de capacidad no utilizada se manifieste en un mayor nivel relativo de los coeficientes capital/trabajo. Es probable además que estas industrias aparezcan con una limitada capacidad de generación de empleo debido a su limitado grado de integración vertical y a su escaso dinamismo.

\section{Consideraciones sobre los problemas de tecnología}

Con el fin de poder evaluar el alcance y las posibilidades reales de las medidas de política encaminadas a promover la selección de tecnologías adecuadas en los países de escaso desarrollo, es pertinente referirse, aunque sea brevemente, a algunas de las explicaciones que se han dado de las causas del atraso científico y tecnológico en estos países.

Entre las explicaciones más comúnmente dadas están: que el atraso se debe a alguna condición inherente en la población de los países atrasados económicamente que los incapacita para el progreso material y los hace desinteresarse de las cuestiones científicas y técnicas; otra explicación se refiere a la reducida asignación de recursos a las actividades de investigación, debido a la incomprensión e ignorancia de los gobiernos, y de la sociedad en general, sobre la importancia estratégica del avance tecnológico. ${ }^{4}$

En otro sentido, se afirma que el uso de tecnologías inadecuadas en los países subdesarrollados se deriva de la falta de factores complementarios, tales como la escasez de capital, personal técnico calificado, capacidad organizativa, etc. Al parecer, todas estas explicaciones se refieren a las manifestaciones visibles más que a las causas originarias del atraso científico y tecnológico.

Una de las formas de aproximarse a la explicación del atraso científico y tecnológico, desde el punto de vista institucional, es a través del examen del papel que han jugado el Estado y los empresarios privados en el proceso de innovación tecnológica. Como es bien sabido, la actividad productiva en los países no desarrollados ha estado históricamente

4 Amílcar O. Herrera, Ciencia y política en América Latina, México, Siglo XXI Editores, 1972, p. 44. 
condicionada por factores y acontecimientos que han operado a escala internacional. En el caso de los países latinoamericanos, el reciente proceso de industrialización, observado a partir de la segunda guerra mundial, ha consistido esencialmente en la sustitución de importaciones a través de la incorporación de tecnologías producidas en el exterior, sin que se hayan necho esfuerzos locales de investigación científica y tecnológica.

El papel del Estado se ha reducido básicamente a promover obras de infraestructura, a levantar barreras proteccionistas y a dictar medidas que tienden a disminuir el riesgo y a aumentar la redituabilidad de las inversiones privadas. Hasta ahora la mayoría de los gobiernos latinoamericanos no han tenido la visión, ni han diseñado los mecanismos para comprometer a las empresas públicas y privadas a desarrollar una capacidad tecnológica propia. Al haberse reducido la función del Estado al apoyo y protección de la actividad económica privada, quedó excluida la posibilidad de cualquier tipo de intento para influir en el tipo de bienes que se producen, $o$ en el tipo de procesos tecnológicos que se adoptan. Las consecuencias de esta situación son bien conocidas: ha habido una creciente extensión horizontal del aparato productivo, la integración vertical de los diferentes sectores económicos ha sido sumamente limitada, y el tipo de tecnología que se ha incorporado no ha sido necesariamente el más adecuado.

Por otro lado, la posibilidad de acceso a la tecnología generada en el exterior ha propiciado una disminución en la presión para desarrollar una capacidad inventiva local. De modo que la incorporación pasiva e indiscriminada de tecnología ha sido uno de los factores que en mayor grado han condicionado el atraso científico y tecnológico en los países de escaso desarrollo.

En las circunstancias actuales no es conveniente que la función del Estado continúe reduciéndose a apoyar incondicionalmente la actividad económica privada; se precisa su intervención más directa en la conducción del proceso económico. En el caso específico de la política tecnológica, dado el desinterés tradicional de los empresarios privados para desarrollar una capacidad tecnológica propia, corresponde al Estado la principal labor de promoción y organización de las actividades de investigación científica y tecnológica. Deben preverse, sin embargo, los mecanismos para lograr la participación del sector privado, ya que no sería conveniente liberar a las empresas de este sector de su responsabilidad en el proceso de innovación tecnológica.

\section{ANÁLISIS EMPf́RICO}

Con el fin de analizar los diversos factores que influyen en la estructura tecnológica y en los niveles de empleo, interesa distinguir dos aspectos importantes: la combinación de productos y la combinación de procesos.

No obstante que la elección de productos y procesos técnicos generalmente se hace en forma simultánea, esta distinción es útil en vista de los diferentes aspectos que influyen, por un lado en la estructura de la producción, y por el otro en el tipo de procesos tecnológicos que se adoptan. Además este procedimiento permite distinguir entre las diferentes implicaciones de política de ambos aspectos dentro de una estrategia de desarrollo industrial y tecnológico. 


\section{La combinación de productos}

En el análisis de la combinación de productos se consideran los efectos de la estructura productiva sobre el empleo, tanto desde el punto de vista directo como indirecto. La idea es que ciertos sectores tienen relativamente más posibilidades de generar oportunidades de empleo que otros, debido a su estructura tecnológica, al diferente grado de dinamismo que los caracteriza, y al mayor o menor grado de integración vertical de sus procesos productivos.

Como primer paso para aproximarse al conocimiento de la capacidad de generación de empleo de las distintas industrias es necesario definir el tipo de tecnología que se asocia con la producción de determinados bienes. Con base en indicadores tales como la relación capital/trabajo y capital/producto se definen tres diferentes estratos tecnológicos. Los indicadores mencionados tienen la limitación de dar una visión estática de las posibilidades de generación de empleo, por lo que se estiman adicionalmente otros indicadores, tales como los coeficientes de elasticidad empleo-producción y empleo-acumulación de capital, y los multiplicadores de empleo.

a) Clasificación de industrias en estratos tecnológicos. La razón para intentar esta clasificación es deducir el grado de intensidad factorial asociado a determinados productos. Dado que no se incluye en el análisis la influencia de la capacidad no utilizada, el término "intensidad factorial" en este caso no se refiere al grado de utilización del capital y el trabajo existentes, sino más bien a la proporción en que estos factores intervienen en el proceso productivo o sea que los niveles de las relaciones capital/trabajo y capital/producto constituyen indicadores de la estructura tecnológica establecida.

Los datos utilizados para definir los tres estratos tecnológicos con base en el nivel relativo de las relaciones capital/trabajo y capital/producto provienen del censo industrial de 1970 (datos preliminares). El concepto de capital corresponde al valor de los activos fijos brutos, el de trabajo al número de personas ocupadas, y el de producto al valor agregado censal, habiéndose trabajado a un nivel de desagregación de tres dígitos.

Como puede observarse en el cuadro 1, las industrias se clasificaron en tres categorías, según el nivel de sus coeficientes capital/trabajo y capital/producto. Se consideraron de nivel bajo (B) las que tienen un nivel inferior al $80 \%$ del promedio de manufacturas; de nivel intermedio (I) aquellas con coeficiente que fluctúan hasta en $20 \%$ alrededor del promedio; y de nivel alto (A) aquellos coeficientes con un valor superior al $20 \%$ del promedio en manufacturas.

Es de interés notar que varios grupos industriales caen dentro de un mismo estrato al considerar los dos criterios de clasificación. O sea que hay industrias en las que tanto la relación capital/trabajo como la relación capital/producto son bajas. Esta situación puede interpretarse como reflejo de la capacidad potencial de estas industrias para generar ocupación directa, ya que aparecen con indicadores de una mayor utilización de mano de obra (baja relación capital/trabajo) y con indicadores que reflejan una utilización relativamente eficiente del capital instalado 


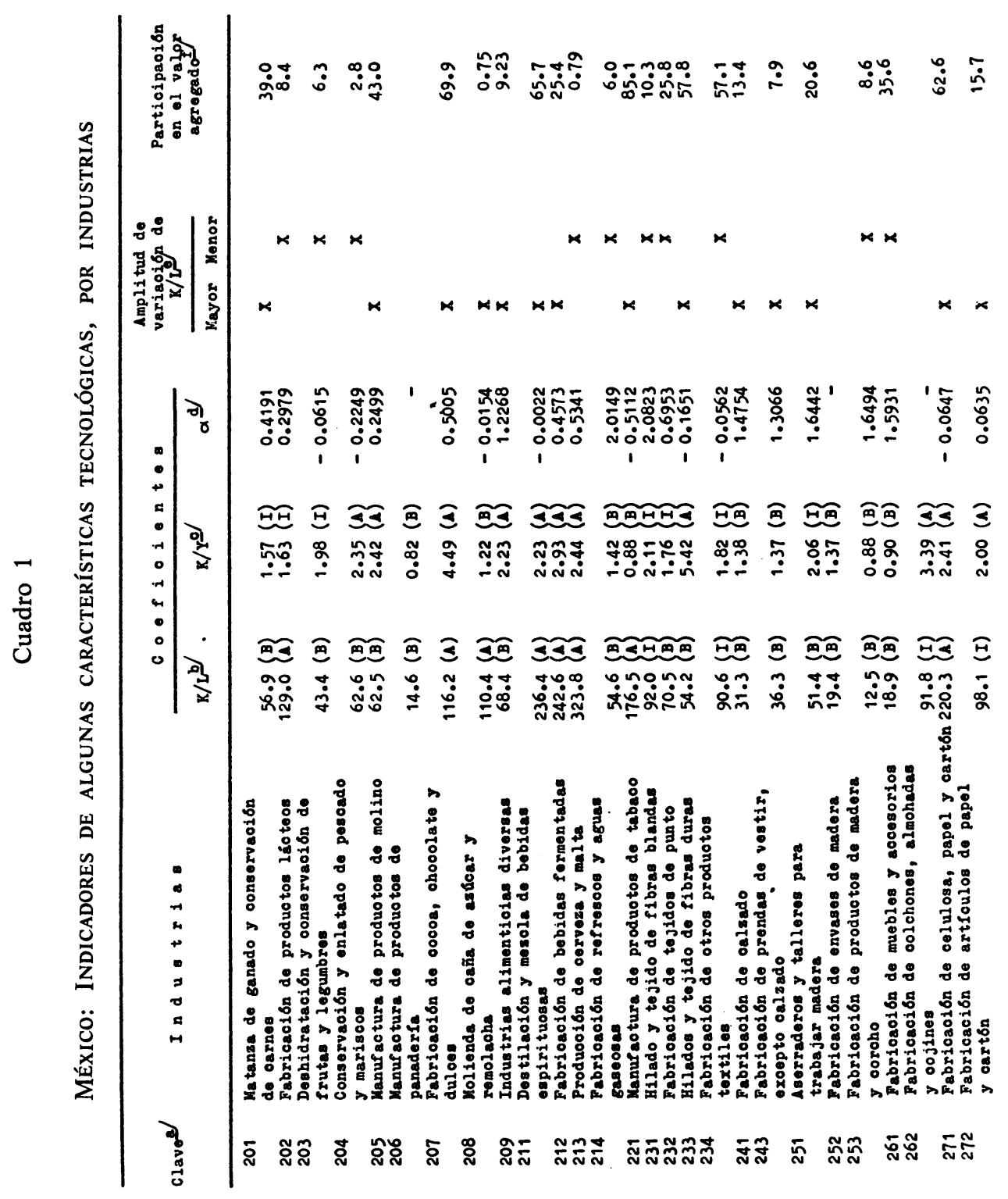




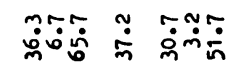

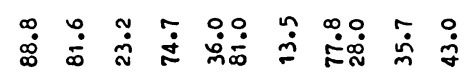

$\stackrel{5}{\dot{m}}$

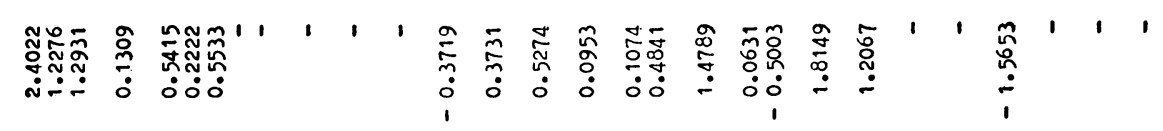

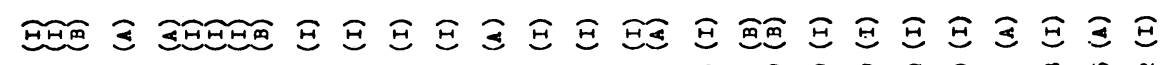

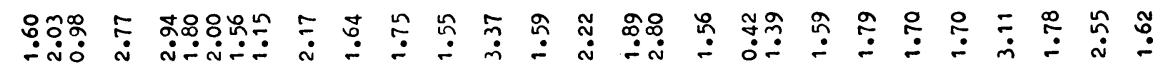

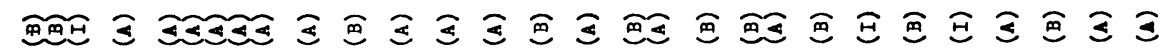

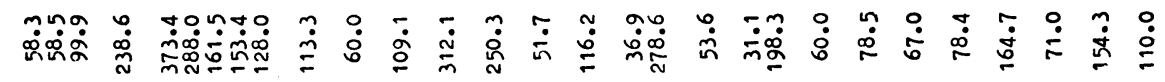

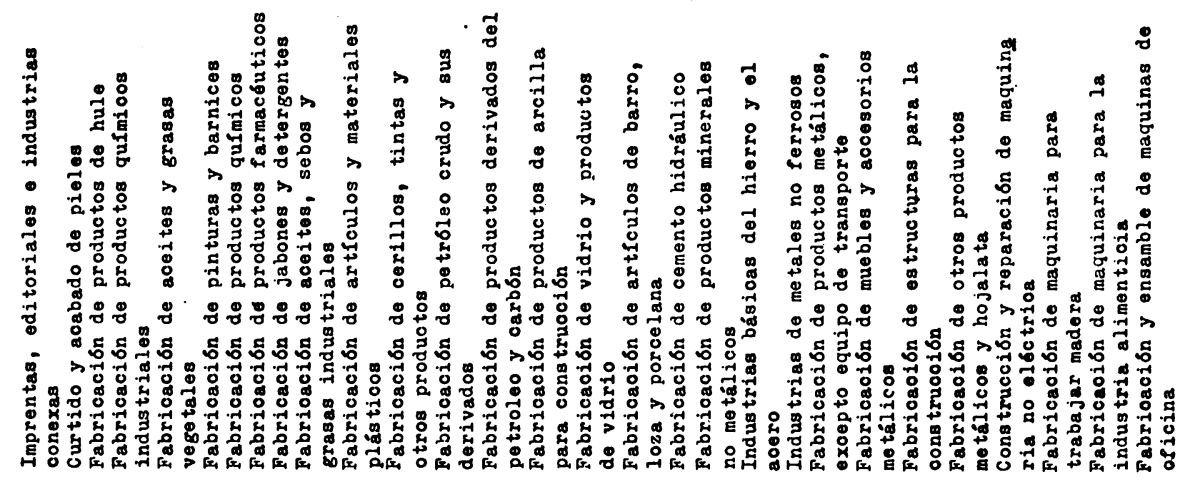

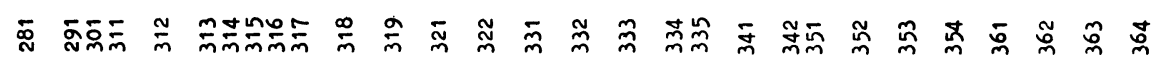




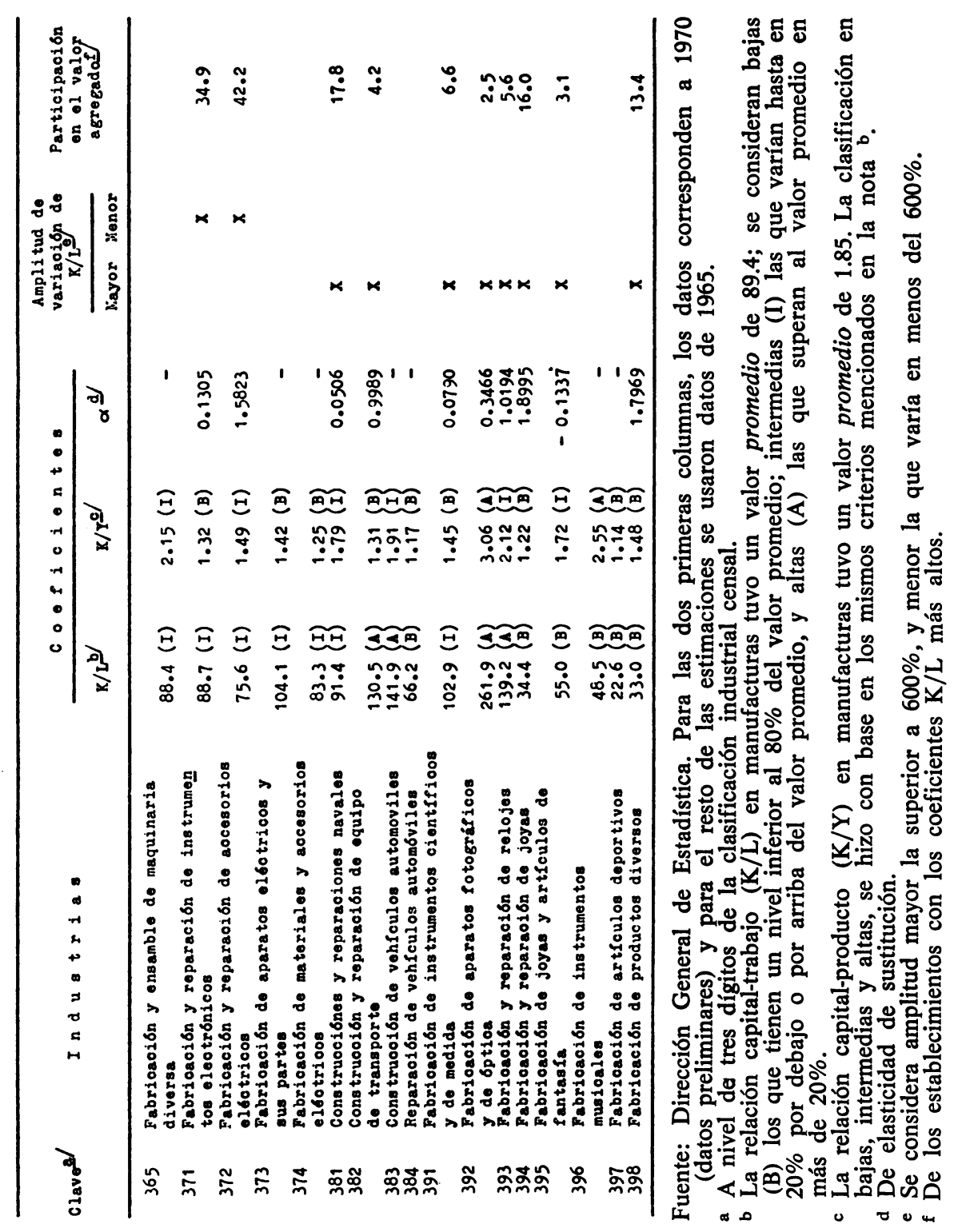


(bajas relaciones capital/producto). Dentro de tales industrias cabe mencionar las de productos de panadería y pastelería, tejidos de punto, fabricación de muebles, industrias del cuero, fabricación de productos metálicos, construcción y reparación de maquinaria, aparatos accesorios y artículos eléctricos, y construcción de equipo ferroviario.

En el caso de las industrias que caen en distintos estratos según se use uno $\mathrm{u}$ otro criterio, tal situación puede ser reflejo del diferente grado de eficiencia de estas industrias o de características tecnológicas específicas. Por ejemplo, las industrias que aparecen con relaciones capital/trabajo relativamente menores que sus relaciones capital/producto, posiblemente son industrias de productividad deficiente o que operan con una proporción considerable de capacidad no utilizada. Algunas de las industrias que caen en este grupo son: fabricación de textiles, manufactura de productos alimenticios, elaboración de bebidas, industrias de la madera, e imprentas, editoriales e industrias conexas.

Por otro lado, en el caso de las industrias que aparecen con coeficientes capital/trabajo y capital/producto relativamente mayores, posiblemente se trata de industrias cuyos procesos tecnológicos se caracterizan por un mayor grado de intensidad de capital. Dentro de este caso están molienda de caña de azúcar, remolacha y su refinación, producción de cerveza, fabricación de celulosa y papel, productos químicos, derivados del petróleo, fabricación de cemento, e industrias básicas del hierro y el acero. En general, casi todas estas industrias se caracterizan por ser de procesos continuos, y por tener un cierto grado de inflexibilidad tecnológica.

La clasificación de las industrias con base en los coeficientes capital/trabajo y capital/producto constituye apenas una primera aproximación al conocimiento de las características tecnológicas de cada industria. Además, en la interpretación de los coeficientes deben tenerse en cuenta las limitaciones derivadas de que los factores capital y trabajo se consideran homogéneos, así como el problema de que se soslaya la influencia de la capacidad no utilizada de tal modo que los indicadores no constituyen sino una primera visión de la capacidad para absorber mano de obra en las distintas industrias.

Con el fin de examinar las posibilidades de generación de empleo desde el punto de vista dinámico, se consideró útil hacer estimaciones de las elasticidades y multiplicadores de empleo asociados a los distintos sectores.

b) Elasticidades de empleo. Se estimaron dos tipos de elasticidad, la de empleo-producción y la de empleo-acumulación de capital. Los coeficientes de elasticidad miden la relación entre las tasas de crecimiento del empleo con respecto al crecimiento de la producción y los acervos de capital. Tanto el nivel de agregación como el período de cobertura estuvieron determinados por la disponibilidad de información estadística.

La principal dificultad para hacer estimaciones a partir de los datos censales se derivó de la carencia de datos sobre capital a precios constantes, de modo que se utilizaron los datos de acervo de capital presentados en las cuentas nacionales. ${ }^{5}$ Para el concepto de trabajo se utili-

5 Cuentas nacionales y acervos de capital, 1950-1967, Banco de México, Departamento de Estudios Económicos, México, 1969. 
zaron las estimaciones de fuerza trabajo, por ramas, de la matriz de insumo-producto. Dado que estas cifras a nivel de las 45 ramas de la matriz se consideraron poco confiables, se procedió a agregar toda la información a nivel de quince sectores. En esta forma muchas de las inexactitudes a nivel de las 45 ramas se eliminan parcialmente. Por razones de consistencia se utilizaron los datos del producto interno bruto a precios de 1960 de las cuentas nacionales.

Las elasticidades de empleo son un reflejo de las repercusiones sobre la ocupación derivadas del diferente dinamismo de la producción y de la capitalización. Sin embargo, tiene la limitación de que se refieren a períodos pasados, es decir, son simples indicadores de tendencia, que reflejan las diferentes tasas de crecimiento relativo de los conceptos involucrados.

Los resultados obtenidos se presentan en el cuadro 2. Es interesante observar que varias de las actividades que aparecían como más intensivas en mano de obra no destacan especialmente por su generación de empleo a través del tiempo. Tal es el caso de las industrias del calzado y de productos de piel, fabricación de muebles, elaboración de productos alimenticios y fabricación de textiles, las cuales aparecen con coeficientes de elasticidad empleo-acumulación de capital relativamente bajos.

En contraste, el sector de fabricación de productos metálicos aparece con indicadores de una mayor utilización de mano de obra, tanto desde el punto estático como dinámico. Al nivel de agregación al que se estimaron los coeficientes de elasticidad, lo más razonable es subrayar la existencia de ciertos indicios de que las industrias de productos metálicos tienen una mayor capacidad de generación de empleos. Cabe señalar además que en el sector de fabricación y reparación de productos metálicos están incluidas cinco diferentes ramas de la matriz de insumoproducto: construcción y reparación de maquinaria, construcción y reparación de aparatos accesorios y artículos eléctricos, construcción de equipo de transporte, ensamble de automóviles, e industrias manufactureras diversas. De modo que es razonable esperar que las posibilidades de generación de empleo varíen entre estas industrias.

Los otros sectores con coeficientes de elasticidad superiores a la unidad son construcción y servicios lo cual se explica en parte por el mayor crecimiento relativo de la mano de obra que ha caracterizado a estos sectores en épocas recientes.

c) Multiplicadores de empleo. El nivel de los multiplicadores ocupacionales, o de empleo, es un indicador del grado de integración vertical de los distintos procesos productivos. El mayor grado de desarrollo económico general se refleja en el grado de complementariedad interindustrial y en el mayor eslabonamiento interno de la producción de bienes de consumo, intermedios y de capital. En este sentido, uno de los objetivos más importantes del desarrollo económico es fortalecer las relaciones intersectoriales y lograr que la producción de bienes finales esté integrada verticalmente dentro del país.

Para la estimación de los multiplicadores de empleo es necesario contar con los coeficientes técnicos de la matriz de insumo producto. Dado que aún no se elabora la matriz para 1970, a partir de los datos de 1960 se estimaron los coeficientes para 1970 utilizando la matriz incremental. El nivel de agregación que se utilizó fue el de los quince sectores de 


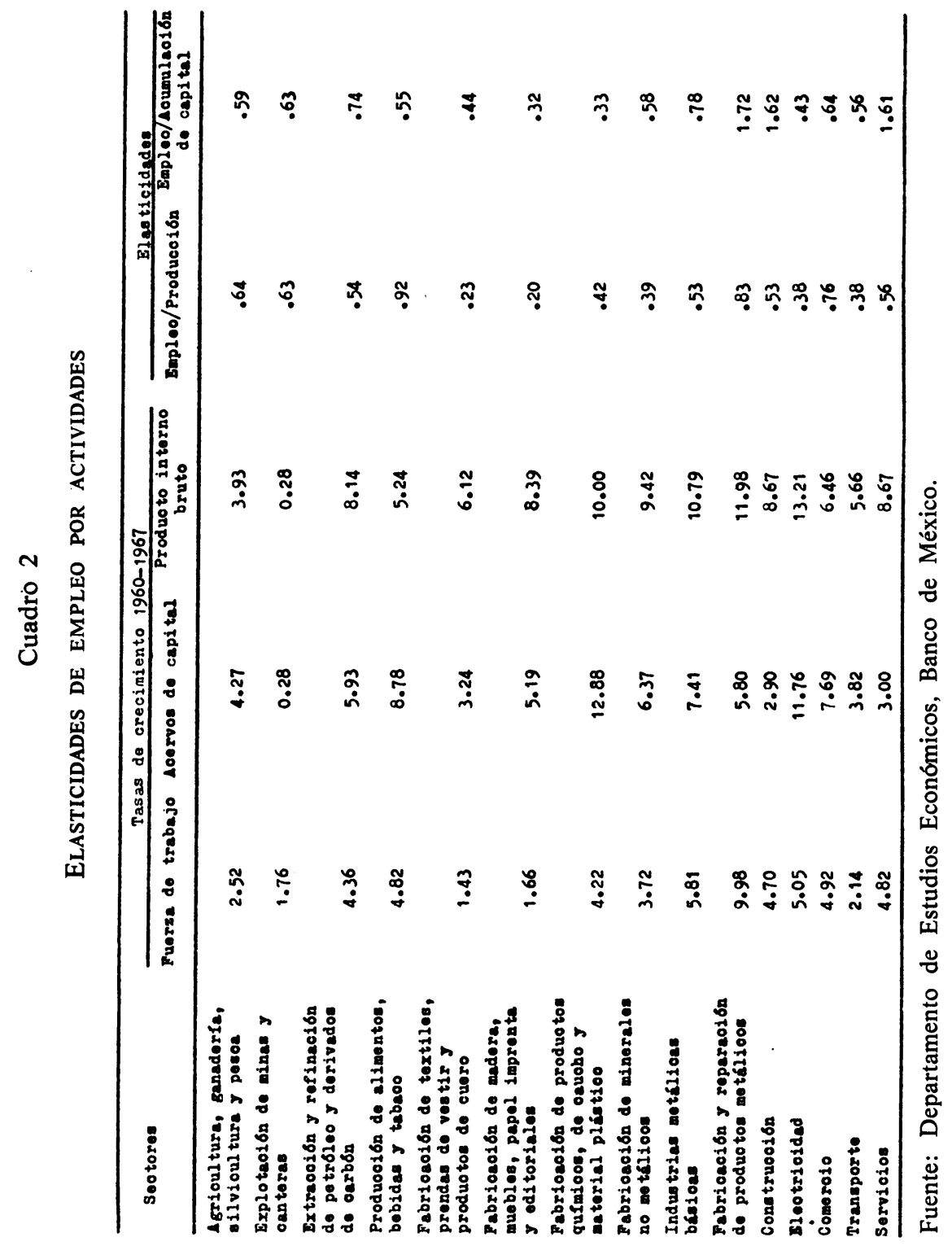


actividad económica. Las etapas seguidas para la estimación de los multiplicadores de empleo fueron:

i) Obtención de la matriz de requisitos directos e indirectos por unidad de demanda final.

ii) Con base en los datos de ocupación y el valor bruto de la producción en cada sector se calculan los coeficientes de ocupación en términos de hombres-año por unidad de la producción.

iii) Se multiplica la matriz de requisitos directos e indirectos por los coeficientes de ocupación respectivos, obteniéndose la ocupación total por unidad de demanda final.

iv) Los multiplicadores se calculan dividiendo la ocupación total entre la ocupación directa en cada sector económico.

Los resultados obtenidos se presentan en el siguiente cuadro:

\section{Cuadro 3}

MÉXico: Multiplicadores DE EMPLEO, POR SECTORES, 1970

\begin{tabular}{|c|c|c|c|}
\hline Sectores & total $\begin{array}{l}\text { Empleo } \\
(1)\end{array}$ & $\begin{array}{c}\text { Empleo } \\
\text { directo generado } \\
\text { (2) }\end{array}$ & $\begin{array}{c}\text { Multiplicadores } \\
\text { de enpleo } \\
(1) /(2)\end{array}$ \\
\hline $\begin{array}{l}\text { Agricultura, ganadería, } \\
\text { slvicultura, caza y pesca }\end{array}$ & .049647 & .041589 & 1.19 \\
\hline Beplotación de minas y canteras & .028214 & .016688 & 1.69 \\
\hline $\begin{array}{l}\text { Extracción y refinación de } \\
\text { petróleo y derivados de carbón }\end{array}$ & .009371 & :004117 & 2.28 \\
\hline $\begin{array}{l}\text { Productos alimenticios, bebidas } \\
\text { y tabaco }\end{array}$ & .030752 & .003267 & 3.72 \\
\hline $\begin{array}{l}\text { Fabricación de textiles, prendas } \\
\text { de vestir } y \text { productos de cuero }\end{array}$ & .034094 & .017817 & 1.91 \\
\hline $\begin{array}{l}\text { Fabricación de madera, muebles, } \\
\text { papel imprenta y editoriales }\end{array}$ & .045825 & .022637 & 1.72 \\
\hline $\begin{array}{l}\text { Fabricacion de productos quimicos } \\
\text { de caucho y material plastico }\end{array}$ & .023110 & .007731 & 2.98 \\
\hline $\begin{array}{l}\text { Fabricación de minerales no } \\
\text { metálicos }\end{array}$ & .034207 & .022809 & 1.50 \\
\hline Industria metálicas básicas & .036541 & .019430 & 1.88 \\
\hline $\begin{array}{l}\text { Fabricación y reparación de } \\
\text { productos metálicos }\end{array}$ & .028951 & .014316 & 2.07 \\
\hline Construcción & .033554 & .018414 & 1.88 \\
\hline Electricidad & .013027 & .007880 & 1.78 \\
\hline Comercio & .015310 & .011281 & $1 \cdot 36$ \\
\hline Transporte & .033263 & .027516 & \\
\hline Servicios & .041439 & .035546 & 1.17 \\
\hline
\end{tabular}

a Por unidad de producción.

Entre los sectores que destacan por tener multiplicadores de empleo relativamente elevados están: productos alimenticios, bebidas y tabaco; fabricación de productos químicos, caucho y material plástico; extracción y refinación de derivados del petróleo y carbón; y fabricación y reparación de productos metálicos.

Los multiplicadores altos pueden reflejar un mayor grado de integra- 
ción de los procesos, o bien que buena parte de los insumos provienen de sectores que utilizan una proporción elevada de mano de obra. En el caso de las estimaciones anteriores no es posible distinguir con precisión el origen de los multiplicadores elevados, aunque probablemente su alto nivel se debe primordialmente a que utilizan insumos con una mayor intensidad de mano de obra, más que a efectos derivados de "insumos" de capital. Un argumento adicional a favor de esa explicación es que los multiplicadores estimados se refieran al plazo corto en el cual el flujo de los insumos por concepto de capital fijo, a diferencia del flujo de insumos de materias primas y productos intermedios, no es simultáneo con el flujo de los productos finales que contribuyen a fabricar. Para apreciar los efectos ocupacionales indirectos derivados de la demanda de bienes de capital habría que considerar períodos relativamente más largos. Esta limitación no cambia sustancialmente los resultados en el caso de los países de escaso desarrollo, dado que los insumos de capital fijo en su mayoría provienen del exterior.

En general, los niveles de los multiplicadores estimados son bastante bajos. En Estados Unidos, por ejemplo, el nivel del multiplicador ocupacional en alimentos, bebidas y tabaco es de 9.3; en productos químicos de 7.1, y en productos derivados del petróleo y carbón de $13.8 .^{6}$

\section{La combinación de procesos}

El objetivo central del análisis de la combinación de procesos tecnológicos es obtener mayor conocimiento de las posibilidades de sustitución factorial, entendida como la sustitución de capital por mano de obra. Este conocimiento es pertinente para el diseño de una política encaminada a influir en la estructura tecnológica.

Pero hay pocos estudios que muestren en forma precisa y sintética la posibilidad de alternativas tecnológicas. Los resultados del presente análisis y los indicadores de flexibilidad tecnológica tienen carácter provisional. La información disponible se redujo en esta etapa a los datos censales, los cuales involucran un nivel de agregación que impide tener un conocimiento preciso de la estructura tecnológica de las distintas industrias.

Por otra parte, la localización de sectores con posibilidades de sustitución factorial no implica necesariamente que tal sustitución sea conveniente desde el punto de vista de los costos o desde el punto de vista de la generación de empleos. En realidad la promoción de tecnologías con mayor intensidad de mano de obra no puede ser indiscriminada, ni puede ser un objetivo general de largo plazo.

Por el momento no es posible entrar en la discusión de la conveniencia de utilizar tecnologías más tradicionales, intensivas en mano de obra, en los países con abundancia de este factor. En todo caso conviene aceptar que la utilización de tecnologías "más manuales" no puede constituir un objetivo en sí mismo, ya que, después de todo, el crecimiento económico es una consecuencia del mejoramiento tecnológico y de au-

6 Véase "Consideraciones sobre la ocupación industrial", Cuadernos del ILPES, Núm. 8, Santiago de Chile, 1969. 
mentos de la productividad. Lo anterior sugiere que el problema tecnológico en los países atrasados económicamente no se reduce sólo a la selección de técnicas que permitan el uso de los recursos y factores disponibles sino también, y principalmente, a la transformación de estos recursos y factores, en forma que sea posible su utilización más productiva. Aceptando que hay una discrepancia entre la dotación de recursos y factores locales, por un lado, y las técnicas que se utilizan, por el otro, el objetivo sería lograr la modificación en ambos polos del problema, de modo que sea posible aproximarlos y lograr la combinación tecnológica más conveniente a los objetivos de desarrollo económico y social.

En el análisis empírico se intenta la localización de sectores donde parezca factible la sustitución factorial. Para esto se utilizan dos indicadores: a) las elasticidades de sustitución $(\sigma)$; y b) la amplitud de variación de la relación capital/trabajo entre empresas de diferente tamaño que pertenecen a un mismo sector industrial.

a) Elasticidades de sustitución. La estimación de las elasticidades de sustitución se hizo con el fin de obtener un primer indicador de la relación existente entre el cambio en las proporciones factoriales y los cambios en el costo relativo de los factores.

El argumento usado para relacionar ambos conceptos consiste en que dados dos factores de producción, capital y trabajo, y de acuerdo con la ley de los rendimientos decrecientes y la ley de proporciones variables, ceteris paribus, a medida que la relación capital/trabajo $(K / L)$ aumenta, la productividad marginal del capital $(P M K)$ decrece, y la productividad marginal del trabajo $(P M L)$ aumenta. Suponiendo condiciones de equilibrio, bajo competencia perfecta y maximización de utilidades, $P M K=r$ (la tasa de utilidades) y $P M L=w$ (la tasa de salarios). Por lo tanto, la elasticidad de sustitución se define como:

$$
\sigma=\frac{\frac{d(K / L)}{K / L}}{\frac{d(w / r)}{w / r}}
$$

El denominador de la expresión anterior equivale a la tasa marginal de sustitución (TMS) entre trabajo y capital. Debido a que la TMS decrece a medida que aumenta la sustitución de trabajo por capital (o sea que la relación $K / L$ aumenta), la elasticidad de sustitución será siempre positiva.

El nivel de la elasticidad de sustitución puede interpretarse como la facilidad para sustituir trabajo por capital. Cuando es alta, significa que hay mayor similitud entre los factores productivos. Mientras que cuando es baja, los factores son muy diferentes entre sí, y por lo tanto es más difícil que se sustituyan entre ellos mismos.

Adicionalmente, se supone que ceteris paribus, el nivel de la elasticidad de sustitución tiene una influencia importante en la distribución funcional del ingreso. Un aumento de la relación capital/trabajo estará acompañada de un aumento, disminución o constancia de la participa- 
ción del capital en el ingreso, dependiendo de que la elasticidad de sustitución sea mayor, menor o igual a la unidad.

Es fácil entender este razonamiento recordando la fórmula de la elasticidad de sustitución. En el numerador se representa la variación de $(L / K) \mathrm{y}$, en el dominador la variación $w / r ; \sigma>1$, significa que el aumento relativo de $K / L$ es mayor que el aumento de $w / r$ y por lo tanto habrá un aumento en la participación del capital; $\sigma<1$, implica que el aumento relativo de $K / L$ es menos que proporcional al aumento de $w / r$ y por lo tanto habrá una menor participación del capital en el ingreso; $\sigma=1$, significa que hay cambios proporcionales en $K / L$ y $w / r$ y la distribución del ingreso permanece constante. Este es el caso de la función de producción Cobb-Douglas.

Lo anterior implica el supuesto de que la distribución funcional del ingreso está determinada por el nivel del coeficiente de elasticidad de sustitución. Es posible, por consiguiente, relacionar la participación de los salarios (o de las utilidades) en el ingreso total con los cambios en la relación capital/trabajo, y estimar el nivel de coeficiente $(\alpha)$, que podría interpretarse como un indicador de la elasticidad de sustitución. Para esto se estimó a través de logaritmos la siguiente regresión:

$$
\frac{W L}{V A}=\beta\left(\frac{K}{L}\right)^{\alpha}
$$

donde:

$$
\begin{aligned}
\frac{W L}{V A} & =\text { participación de los salarios en el valor agregado. } \\
K / L & =\text { relación capital/trabajo. } \\
\alpha & =\text { coeficiente de "elasticidad de sustitución". } \\
\beta & =\text { término independiente. }
\end{aligned}
$$

Las observaciones utilizadas se refieren a los establecimientos de diferente tamaño de las industrias incluidas en el Censo Industrial de 1965 a nivel de tres dígitos. De acuerdo con los supuestos, el mayor nivel relativo del coeficiente $(\alpha)$ sugiere la existencia de mayores posibilidades de sustitución factorial. Deben tenerse en cuenta, sin embargo, las limitaciones derivadas del método de estimación y de la serie de supuestos implícitos, de modo que los resultados no pretenden ser sino indicadores parciales de flexibilidad tecnológica, cuya corroboración deberá sujetarse a investigaciones posteriores. En la tercera columna del cuadro 1 se presentan los resultados de las estimaciones del coeficiente de "elasticidad de sustitución" en las distintas industrias.

Podría afirmarse que los coeficientes con un nivel superior a 1.51 se asocian con industrias donde parece existir una gama de alternativas tecnológicas, tales como las industrias textiles, fabricación de prendas de vestir, fabricación de productos de madera, industrias metálicas, y fabricación de aparatos y accesorios eléctricos. En tanto que los coeficientes con un menor nivel relativo se asocian con industrias como: producción de cerveza, fabricación de artículos de papel y cartón, fabricación de derivados del petróleo y del carbón mineral, fabricación de vidrio e industrias químicas. 
En términos generales, éstas son industrias en las que el tipo de procesos tecnológicos que utilizan parece ser relativamente más específico.

b) Amplitud de variación de las relaciones capital/trabajo. Otro de los indicadores utilizados para detectar posibilidades de flexibilidad tecnológica fue el grado relativo de variación de los coeficientes capital/trabajo. En los casos en que el margen de variación es amplio, las diferencias pueden atribuirse a la utilización de distintas técnicas, mientras que cuando los márgenes de variación son relativamente menores indicarían la existencia de escasas alternativas tecnológicas. El supuesto subyacente es que la existencia de diferentes niveles de los coeficientes capital/trabajo es, en cierto modo, evidencia de que existen alternativas tecnológicas dentro de un mismo sector industrial debido a la influencia de economías a escala. Una limitación adicional que merece tenerse presente es el supuesto de que la producción proveniente de los distintos establecimientos dentro de un mismo grupo industrial es homogénea.

El método seguido para estimar los márgenes de variación tecnológica en cada industria fue el siguiente: como primer paso, se estimó la variación porcentual entre los niveles máximo y mínimo de los coeficientes capital/trabajo para los establecimientos de diferente tamaño pertenecientes a las industrias clasificadas a nivel de tres dígitos en el Censo Industrial de 1965. El criterio de tamaño de los establecimientos que se utilizó fue el número de personal ocupado. Una vez estimada la amplitud de variación relativa de los coeficientes capital/trabajo, se procedió a identificar a las distintas industrias, según el margen de variación relativo de sus coeficientes; se consideró que las industrias con tecnologías relativamente más homogéneas son aquellas en las que el coeficiente capital/trabajo tiene una amplitud de variación inferior al $600 \%$; y que las industrias con mayor "heterogeneidad" tecnológica aquellas en las que el margen de variación de las relaciones capital/trabajo fue superior al $600 \%$ (véase el cuadro 1).

El mayor margen de variación de los coeficientes capital/trabajo refleja la posible existencia de una mayor gama de alternativas tecnológicas dentro de las industrias. Sin embargo, para tener una visión más precisa de las posibilidades reales de usar tecnologías alternativas, es importante conocer el tipo de tecnología que predomina en cada industria. El conocimiento de este último aspecto es útil, en vista de que puede haber industrias donde la mayor amplitud de variación produzca una imagen falsa sobre la gama de alternativas tecnológicas existentes debido a problemas derivados del nivel de agregación del análisis a tres dígitos, o de la forma de captación de la información censal.

El tipo de tecnología predominante en cada industria tiene una estrecha conexión con el grado de concentración industrial. Como indicador de este último aspecto, se consideró el porcentaje de participación de los establecimientos más intensivos en capital dentro del valor agregado de cada grupo industrial (véase el cuadro 1). En la medida en que es mayor la participación de los establecimientos más intensivos en capital, menores parecerían ser las posibilidades de usar tecnologías alternativas dentro de cada tipo de industria, en especial cuando las características tecnológicas de ese grupo industrial presentan cierto grado de homogeneidad.

De acuerdo con los resultados estimados, las industrias donde parece 
más viable la utilización de diversas alternativas tecnológicas son aquellas donde la amplitud de variación de los coeficientes capital/trabajo es mayor, y la participación de los establecimientos más intensivos en capital dentro del valor agregado es relativamente menor. Dentro de esta categoría están las industrias alimenticias diversas, fabricación de calzado, industrias del cuero, construcción y ensamble de equipo de transporte y de vehículos automotrices.

Por otro lado, el mayor grado aparente de uniformidad tecnológica, reflejado en el reducido margen de variación de los coeficientes capital/trabajo en ciertas industrias no es necesariamente reflejo de inflexibilidad tecnológica. Al nivel de agregación que se trabajó, se confunden dentro de un mismo grupo industrial actividades que a menudo son complementarias. La imposibilidad de distinguir entre el proceso tecnológico central y los procesos periféricos impide examinar con mayor precisión las posibilidades de utilizar tecnologías alternativas, que permitan una mayor absorción de mano de obra.

A partir del análisis de la amplitud de variación de los coeficientes capital/trabajo sólo es posible hacer algunas aproximaciones al conocimiento del tipo de tecnología que predomina en cada grupo industrial. Dichas aproximaciones tienen un carácter tentativo y deberán de complementarse con análisis mucho más detallados, a nivel más desagregado.

\section{CONSIIDERACIONES FINALES}

Las posibilidades de hacer una selección tecnológica adecuada en los países en desarrollo dependen de que exista cierta capacidad para decidir acerca de los bienes que se han de producir y las técnicas que más conviene adoptar. En el caso de los países de menor desarrollo es esencial lograr la mayor coherencia entre los objetivos de aumentar la producción masiva de bienes para el consumo interno, promover la especialización de ciertas ramas industriales, aumentar el grado de integración vertical de los procesos productivos, aumentar las exportaciones y generar un mavor número de oportunidades de empleo.

En este sentido la política referente a la combinación de productos ha de concebirse como una política de promoción y "contención" selectiva de las distintas industrias. En la selección de productos deben considerarse dos criterios: la relación entre tales productos y las necesidades de la población, y las posibilidades de integrar localmente la producción de los bienes finales. La utilización de estos criterios permitiría limitar la innecesaria extensión horizontal del aparato productivo y la operación de ciertas industrias por debajo de su capacidad instalada.

En relación con el objetivo de aumentar las oportunidades de empleo, la promoción selectiva de industria no puede reducirse a promover la expansión de sectores con tecnologías más intensivas en trabajo. En el análisis empírico quedó manifiesto que hay sectores que aparecen con indicadores de alta intensidad de mano de obra, pero que no destacan especialmente por su capacidad de generación de empleos, debido en parte a su escaso dinamismo y en parte al limitado grado de integración de sus procesos productivos.

Para la selección de procesos tecnológicos más adecuados a las condiciones de los países en desarrollo se han sugerido una variedad de 
medidas que no son excluyentes sino complementarias. Se ha enfatizado la influencia del sistema de precios e incentivos en la elección de técnicas, y en la dirección de las innovaciones tecnológicas. Sin embargo, cuando la principal fuente de conocimiento tecnológico es de origen importado, como es el caso de los países en desarrollo, la reducción del margen de elección es evidente y el sistema de incentivos locales pasa a tener una importancia secundaria. Hay evidencia empírica que muestra que el tamaño o escala de operación tiene una influencia crucial en la selección de técnicas en algunas industrias. ${ }^{7} \mathrm{De}$ manera que el objetivo en esta área se reduce a procurar que el sistema de incentivos se mueva en una direcciónsconsistente con los objetivos tecnológicos, sin olvidar que esta condición dista de ser suficiente para influir de una manera definitiva en la elección de tecnologías.

Es de mayor importancia aumentar la capacidad de investigación y desarrollo experimental como medio para aumentar la gama de alternativas tecnológicas. En la mayoría de los países en desarrollo es evidente el escaso esfuerzo de investigación relacionado con la industria, de manera que la promoción de investigación aplicada seguramente tendrá una influencia positiva. El simple establecimiento de metas generales de gasto puede ser insuficiente si no se pone especial atención en la dirección y el contenido de la investigación tecnológica. La necesidad de aumentar las posibilidades de empleo de los recursos y factores locales implica diferencias sustanciales en los objetivos de investigación tecnológica en los países subdesarrollados con respecto a los países industrializados. Los resultados del análisis empírico constituyen apenas una aproximación a la localización de posibles alternativas tecnológicas ya existentes dentro de cada grupo industrial. Las mayores perspectivas probablemente están en la generación y el diseño de nuevos procesos técnicos, y en la adaptación parcial de algunos de los procesos ya existentes.

En la parte inicial se hacía referencia a los agentes institucionales que favorecerían el proceso de innovación tecnológica en los países en desarrollo. Se mencionaba el papel central que corresponde al Estado en la labor de promoción y organización de las actividades de investigación científica y tecnológica. Sin embargo, aceptada la importancia de esta labor de organización, existe el peligro de que el proceso de innovación tecnológica solamente esté promovido "desde arriba", con el resultado de que buena parte de los conocimientos e ideas generados en los institutos de investigación no alcancen una aplicación concreta en el proceso productivo, debido a la deficiente demanda de estos servicios.

Esto nos lleva a la consideración de la importancia de contar con el ambiente y las actitudes que favorezcan el proceso de innovación tecnológica entendida como la capacidad para mirar lo viejo en una forma nueva. ${ }^{8}$ Cuando las condiciones económicas y sociales operan a favor del dinamismo tecnológico, las posibilidades de obtener resultados concretos a partir de la labor de promoción de investigación tecnológica aumentan considerablemente. El problema es que la introducción de innovaciones implica un cambio de actitud difícil de institucionalizar. Se

7 Véanse por ejemplo, F. Stewart, "The Choice of Techniques: a Case Study of the Manufacture of Cement in Kenya", Mimeo., 1971, y G. K. Boon, Factores físicos y humanos en la producción, México, Fondo de Cultura Económica, 1970.

8 R. N. Nelson, The Technology Gap and National Science Policy, Growth Center, Yale, Discussion Paper, mayo de 1970. 
necesita considerar que la tecnología extranjera no es un producto terminado, sino una fuente de ideas susceptibles de adaptarse a las condiciones locales y de concretizarse en el diseño de nuevos productos y procesos. Se requiere además la disponibilidad de mano de obra calificada y de un sector de bienes de capital de relativa importancia. ${ }^{9}$

La serie de medidas requeridas para fortalecer la capacidad científica y tecnológica de los países en desarrollo forma parte necesariamente de un conjunto de políticas orientadas a influir en aspectos tales como las actividades de investigación básica y aplicada, la organización del sector público, la estructura industrial, los patrones de demanda, la participación del capital extranjero en el proceso de producción y distribución, la formación de recursos humanos y el adiestramiento de la mano de obra y, en general, en los patrones culturales y las actitudes de la población hacia el proceso de avance científico y tecnológico.

9 H. Pack y M. Todaro, "Technological Transfer, Labour Absorption and Economic Development", Oxford Economic Papers, noviembre de 1969. 